\title{
Methodological approach for the evaluation of FOXO as a positive regulator of antioxidant genes
}

\author{
María Monsalve ${ }^{1^{*}}$, Ignacio Prieto ${ }^{1}$, Andreza Fabro de Bem², Yolanda Olmos ${ }^{3,4}$ \\ ${ }^{1}$ Instituto de Investigaciones Biomédicas “Alberto Sols” (CSIC-UAM). Arturo Duperier 4. \\ 28029-Madrid (Spain). \\ ${ }^{2}$ Florianópolis, SC, Brazil; Institute of Biological Sciences, University of Brasilia, Brasilia, \\ Brazil. \\ ${ }^{3}$ School of Cancer and Pharmaceutical Sciences, King's College London, SE1 1UL London, \\ United Kingdom. \\ ${ }^{4}$ The Francis Crick Institute, NW1 1AT London, United Kingdom ${ }^{4}$ \\ Center of Biological Sciences (CCB), Federal University of Santa Catarina (UFSC), \\ ${ }^{*}$ Corresponding author.
}

\section{Contact Information}

Corresponding author: M.M. (mpmonsalve@iib.uam.es).

Running Head: Keeping FOXO under control. 


\section{Summary/Abstract}

All four FOXO isoforms have been shown to respond to changes in the cellular redox status of the cell, and regulate the expression of target genes that in turn can modulate the cellular oxidative status. However, the mechanisms involved are still controversial. It is clear though that redox regulation of FOXO factors occurs at different levels. The proteins themselves are redox-sensitive and their capacity to bind their target sites seems to be at least partially dependent on their oxidative status. Importantly, several of the cofactors that are known to regulate FOXO transcriptional activity are also sensitive to changes in the cellular redox status, in particular the deacetylase SirT1 is activated in response to reduced levels of reducing equivalents (increased $\mathrm{NAD}^{+} / \mathrm{NADH}^{+}$ratio) and the co-activator PGC-1 $\alpha$ is induced in response to increased cellular oxidative stress. Furthermore, nuclear localization of FOXO factors is also regulated by proteins that, like AKT, are themselves regulated directly or indirectly by the cellular levels of reactive oxygen and nitrogen species. In this technical review, we aim to update the current status of our knowledge of how to handle redox-regulated FOXO factor research in order to better understand FOXO biology.

Key Words: FOXO, vectors, activity, cell culture, pro-survival, metabolism, stress response. 


\section{Introduction}

FOXO factors control critically how the cell responds to metabolic and environmental stressors and channels both pro-survival and pro-apoptotic responses. Therefore, when studying a particular type of FOXO activity is crucial to control adequately the experimental conditions and this is particularly true when analyzing FOXO regulation of oxidative stress resistance [1].

Just as an example, since FOXO activity is tightly regulated constitutively active mutants have been extensively used to analyze FOXO functions in a wide array of biological settings. However, under standard conditions, this will result in the induction of cell death, making it difficult the evaluation of FOXO protective, cell survival-related activities both in vitro and in vivo [2,3].

Most actively-proliferating cells have very limited FOXO levels and activity. Therefore, in order to study FOXO functions the most widely used protocol involves a serum deprivation step to induce cell cycle arrest. However, in sub-confluent and serum deprivation conditions the activation of FOXO normally results in the strong induction of pro-apoptotic pathways and cell death [4]. This pro-apoptotic response can be enhanced by oxidative stress, regardless of whether the source of oxidants is exogenous or endogenous [5].

Therefore, in order to study FOXO pro-survival activities, such as antioxidant gene expression, it is advisable to work in confluent cultures, avoid standard serum deprivation conditions and control tightly the level of FOXO activity.

\section{Materials}

Prepare all solutions using sterilized ultrapure water (prepared by purifying deionized water, to attain a sensitivity of $18 \mathrm{M} \Omega-\mathrm{cm}$ at $25^{\circ} \mathrm{C}$ ) and analytical grade reagents.

Handling of DNA and Viral stocks should be done in a tissue culture hood using sterile pipettes and pipette tips with filter. DNA and viral stocks should be kept at $-80{ }^{0} \mathrm{C}$ for 
long-term storage. DNA should be free of endotoxins and dissolved in ultrapure sterile water.

Viral stocks can be maintained in different solutions, but we generally use cell culture grade, sterile 1xPBS. All viruses loose infectivity upon freezing and thawing. Keep handling time of viruses and DNA at a minimum and keep the stock vials on ice. For viruses, use of screw cap microcentrifuge tubes is recommended, preferably those that have a cap that screws over and not inside the vial, to avoid contamination. Before opening and after closing, a viral vial, spray it with 70\% Ethanol. Work in a bio-safety level 2 tissue-culture hood and follow bio-safety and waste disposal regulations.

Test Cells. To generate adenovirus 293-A cells should be used, to prepare retroviruses and lentiviruses, Phenix cells are a good choice. These two cell types derive from

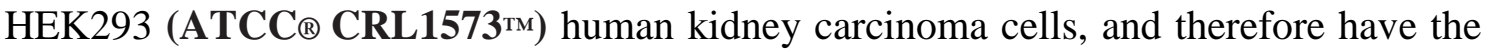
same origin than 293T cells. 293T cells are easy to cultivate (DMEM, 10\% FBS, antibiotics) and are probably the best choice to evaluate protein overexpression/suppression driven by adenoviral/retroviral/lentiviral infection, or plasmid transient transfection because they are easy to transfect/infect, so require low amounts of viruses or DNA. Using adenovirus a moi of 1 is enough to control changes in protein levels with most vectors, the recommended dose for FOXO vectors is 0.2 moi. Split cells 1/4, $48 \mathrm{~h}$ later cells should be confluent. Mix gently the virus with fresh media and add it to the confluent culture. Infection with most vectors is generally done overnight. For FOXO vectors $4-6 \mathrm{~h}$ is recommended in the presence of serum. To evaluate FOXO levels cells can be harvested as early as $12 \mathrm{~h}$ post infection (maximun $24 \mathrm{~h})$.

Target cells of adenoviral infection. The most commonly used adenoviral vectors derive from human adenovirus serotype 5 (HAd5). Targeted cells can vary extensively as reported in the literature. Essentially all cell types of human, mouse, rat and bovine origin can be infected. Lung epitelial cells are the natural target of adenovirus and hence particularly easy to infect. We have extensively used adenovirues to infect human, mouse and bovine endothelial cells (HUVEC, BAEC, MLEC, MAEC), for which the recommended moi is generally 25-50, but for FOXO vectors should be kept bellow 10 . We have also used FAO (rat hepatoma cells) cells and mouse primary hepatocites, they 
require 2-4 less adenoviral levels than endothelial cells. Fibroblast are generally hard to infect and for NIH3T3 cells it is almost impossible. Both confluent and subconfluent cells can be used for Ad infection, preferably in the presence of serum to reduce toxicity and cell death as indicated above. To enhance FOXO pro-cell survival activities after the infection the cells can be incubated in cell culture media without glucose and with 5 $\mathrm{mM}$ galactose in the absence of serum for $48-72 \mathrm{~h}$ or placed in an oxygen controlled cell culture incubator at $3-5 \% \mathrm{O}_{2}$.

Other common reagents that can be used to increase FOXO activity. The most common one is low serum, under 2\% FBS serum this will induce cell death in most cell types, use as an alternative $2 \%$ Horse Serum (HS) is recommended. The second most common reagent used is hydrogen peroxide $\left(\mathrm{H}_{2} \mathrm{O}_{2}\right)$, use of subtoxic doses is recommended. 50 $\mu \mathrm{M}$ for endothelial cells is enough to drive the activation of FOXO, the activated protein can be detected up to $9 \mathrm{~h}$ after the treatment (nuclear, dephosphorylated, deacetylated protein). Nitric oxide (NO), an important regulator of vascular function can also be used to inhibit FOXO activity in endothelial cells. A variety of NO donors can be used induce the phosphorylation of Akt and the inactivation of FOXO, each has an optimun time and dose, we generally used $62 \mu \mathrm{M}$ DETA-NO for $12 \mathrm{~h}$ in serum deprivation conditions on confluent BAEC cultures.

Vectors and mutants. We used the plasmid vectors originally generated by in the laboratory of Dr. K. Walsh and published in [6] to overexpress HA tagged wt FOXO3, and two mutant variants, subcloned into a shuttle vector pAdTrack-CMV, which contains green fluorescent protein (GFP) under the control of a separate cytomegalovirus (CMV). The FOXO3a-AAA triple mutant (TM-FOXO3a) is not phosphorylatable by AKT [7]. The dominant-negative form (dn-FOXO3a) was constructed by deletion of the $\mathrm{C}$ terminus transactivation domain. The shuttle vector was co-transformed into Escherichia coli with the adenoviral backbone plasmid pAdEasy-1 to generate recombinant adenoviral DNA then transfected into the packaging cell line 293A to produce the recombinant adenoviral vectors. The control virus expressing only the GFP transgene (Ad-GFP) was prepared by the same system [8]. Silencing adenoviral vectors wre generated using the pSilencer TM Adeno CMV Promoter System from Ambion. Silencing oligonucleotides were designed and tested by transfection, cloned in pSilencer and transfected into 293A cells to generate viral 
particles. Silencing oligonucleotides and controls were described in [9].

Antibodies. To monitor FOXO protein levels and activity is recommended to test the level of AKT phosphorylation that largely controls FOXO nuclear exclusion. There are a number of good antibodies in the market that are directed specifically against AKT phosphorylation sites. It is also relevant to test the levels of FOXO acetylation that is important in the control of the levels and activity of the protein. Due to the lack of specific antibodies, the protein has first to be immunoprecipitated and then the IP material tested by WB with a second antibody against FOXO and with an anti-Acetyl Lysine antibody. To efficiently IP the FOXO protein even in very stringent conditions like those required for CHIP assays (up to $2 \%$ SDS), the HA-tagged protein can be used since there are very good monoclonal antibodies in the market directed against the HA tag, such as the mouse monoclonal antibody 12CA5. Is also relevant to be able to visualize FOXO sub-cellular localization directly with specific antibodies that can be used in fixed cells and tissues. Finally, when using plasmids or viral vectors that also encode for a reporter protein, like GFP, evaluation of its levels by western blotting can be helpful to estimate the quality of our viral preparation in terms of levels of FOXO/GFP.

\section{Methods}

\subsection{Vectors}

To over-express or knock-down FOXO factors, in principle any kind of vector can be used, however the nature of FOXO activities makes the use of many common strategies somehow difficult, particularly when over-expression of constitutively active mutants is involved.

Transient transfection. Usual transfection protocols require that cells are transfected at $30-70 \%$ confluency in serum deprivation conditions, and after 6 hours to overnight, cells are washed and incubated with regular serum-containing medium with serum prior to analysis 24-72 h post-transfection. Conventional constitutive vectors that drive the over-expression or knock-down of FOXO, specially the constitutively active form, will induce cell cycle arrest and or apoptosis in these conditions. To overcome this problem, 
it is advisable to use either an inducible vector or alternatively a very low copy number of a plasmid that codes for the wild-type construct or expresses it at relatively low levels. In this case, transfections work best when cells are transfected at a relatively high confluency, 70-90\%. To be able to make the transfection work in these conditions the cells have to be split just before transfection. If the transfection reagent requires serum deprivation, transfection time should be reduced to a minimum (4-6 h) $[9,10]$.

Viral vectors. FOXO activity in a virus-infected cell will induce cell death. This is especially true when using constitutive viral vectors but also the inducible ones will be unhappy bearing this gene. Therefore, when a viral stock is prepared that bears a FOXO active gene it can never be assumed that it will always have the same activity, independently of the number of active viral particles. Also, the use of previous viral stocks to generate a new one will result, in most cases, in an important reduction in the level of FOXO activity. Consequently, when working with viral vectors is advisable to always prepare new stocks from scratch, from the viral plasmids. The viral stocks have to be carefully evaluated both for the number of viral particles and the level of FOXO activity and both have to be controlled for in the actual experimental setting.

Another consequence of FOXO activity is that its expression in the packaging cell line used to generate viruses will be detrimental both to the cell and to the virus. Therefore, high levels of transfection (high copy number/cell), low density of the packaging cell in culture and lengthy transfection protocols should be avoided, as noted before in general for transfection protocols. Also, the number of viral particles produced cannot be expected to be very high. In our experience, the average number of particles with FOXO vectors is $10-100$ times lower than with other genes.

Infection of target cells. To achieve an effective integration of the retroviral or lentiviral vector in the target cell genome, viral infection is carried out in subconfluent conditions, and cells are then incubated in media with serum so that they proliferate prior to the selection of positive clones.

Similarly, cell lines that bear retroviral and lentiviral FOXO genes will tend to silence these genes (more than other genes) since they reduce their proliferation rates. Consequently, FOXO activity should never be taken for granted even following 1-2 cell 
passages, and co-expressed gene expression (ie GFP, luc) will not guarantee FOXO activity. Accordingly, the use of inducible vectors would always be strongly recommended, since it will noticeably reduce (not cancel) the problem.

Use of Adenoviral vectors in this context offers and advantage over other gene delivery systems since infections do not require to work in sub-confluency or serum deprivation conditions. Nevertheless, the generation of the viruses themselves has the same limitations as with other viral particles. Similarly, low overexpression rates and use of wild-type over constitutively active FOXOs is highly recommended [9-11].

A standard protocol would be:

Let cells just reach 80-90\% confluency. Reduce serum levels to 2-\%-5\% (depending on cell type to reduce proliferation rate), infect the cells to result in a $2 x$ increase over endogenous FOXO levels for 4-6 h. Change media and let the cells recover o/n with media $+10 \%$ FBS. On the morning cell culture should have just reached confluency. Test FOXO activity.

Additional considerations.

Dosing. Taking into account that the precise control of FOXO activity can be difficult, we rutinarily tested 3 doses in each experimental test. The reference dose would be the one expected to yield the maximum activity, and along with it a 50\% dose and a 2x dose would be tested.

Generation of stable cell lines.

In general, overexpression of FOXO will result in the reduction of cell growth and as indicated, may result in the induction of cell death, particularly in subconfluent cell cultures in the presence of serum. Generally generation of stable cell lines requires the infection with retroviruses or lentiviruses in subconfluent cultures and the cells have to be able to grow and expand later on. Therefore, the use of conditionally activable vectors is highly recommended or when it is not possible, an alternative could be the use of vectors with the wild type protein, not the TM constitutive active version and 
under a relative weak promoter (not like CMV), and avoid tags, that may stabilize the protein. In this conditions overexpression levels will never be very high, but changes in activity may still be significant.

\subsection{Activity assays.}

FOXO proteins are highly regulated at a post-transcriptional level and as a consequence its over-expression or even its silencing does not, guarantee a measurable change in activity. To overcome this problem both constitutively active and negative dominant mutant versions have been developed and widely used [6]. By far the most commonly used version has been the so called TM mutant, for FOXO1 and 3, that carry three point mutations that make the proteins insensitive to inactivation by AKT dependent phosphorylation [12]. The handling of vectors that carry these mutants is particularly difficult since these constitutively active forms effectively drive apoptotic cell death in the cells that express them, including both the packaging cell for viral particles and the target cells. To tackle the problem there are two options. An obvious one is to avoid these mutants, but if that is not possible. There are some considerations that can be helpful.

Dosage. If you intend to evaluate a FOXO activity that is different from cell death it is advisable to reduce the dosage of the protein to the minimum that will provide a read out of the intended determination and that means to work in conditions where overexpression is less than 2x over the endogenous protein.

Timing. FOXO TM mutants do not only activate pro-apoptotic genes even if the obvious endpoint is cell death. Evaluation of the impact of FOXO on other target genes, like stress resistance genes with these mutants is therefore possible before the apoptotic cascade is induced. In general a fully regulated apoptosis process takes $24 \mathrm{~h}$ from the initial signal to the final cell death. If the stimuli is weak (ie low levels of active FOXO) it can take $48 \mathrm{~h}$ or more. That provides a window where other FOXO activities can be evaluated prior to the onset of the apoptotic activities of up to $24 \mathrm{~h}$ but that requires that evaluation quickly follows infection or transfection procedures.

Whenever possible it is better to handle the wild type protein both to increase and 
decrease its activity using wt vectors for its overexpression and sh vectors for its knock down. Over-expression even of the wt protein should be moderate (about 2-fold) and the activity could be exogenously stimulated.

Cell culture conditions.

Confluency. As noted above the cell death-promoting activities of FOXO proteins will be importantly reduced when working with confluent fully cell cycle arrested cultures. As a result, to evaluate FOXO protective roles, it is advisable to work with primary cells or cell lines that effectively respond to confluency and fully arrest cell growth. When that is not feasible it is advisable to work within the time frame in which cells are confluent but not over-confluent.

Serum withdrawal. To enhance FOXO protective activities and to control cell overgrowth it is common to reduce the serum concentration and/or not to use fetal serum to reduce the levels of growth factors. However, growth factor deprivation in standard culture conditions can also result in the activation of apoptotic cell death, in order to prevent it, oxidative metabolism should be activated [5]. This will increase the activity of several FOXO co-factors like PGC-1 $\alpha$ and SIRT1 [11] that cooperate with the FOXO protein to induce protective responses

Glucose/galactose. The easiest way to activate oxidative metabolism is to use a cell media where glucose has been replaced by galactose, this change reduces the glycolytic flux and activates oxidative metabolism. Reduction in the concentration of glucose in the media would have the same effect but can compromise cellular viability. Most nontumor cells when they reach confluency can be maintained in media without serum in the presence of galactose for at least $48 \mathrm{~h}$ without compromising their viability [13].

Oxygen. Most cell incubators do not control oxygen tension, and as a result oxygen concentrations are higher than those encountered by the cells in the organism. Hyperoxia results in the inhibition of oxidative metabolism in order to prevent the resulting oxidative stress, since superoxide production in the mitochondrial ETC increases with supra-physiological oxygen concentrations. Even though each cell type has a different optimal oxygen concentration a good estimate is that incubation in 3-5 \% 
oxygen will result in the activation of mediators of oxidative metabolism [14,15], conditions that result increase FOXO protective roles, preventing the induction of cell death pathways. Under about 2\% oxygen, oxidative metabolism would be compromised.

\subsection{Step by step protocols.}

\subsubsection{Transfection}

A standard protocol would be:

1- Split cells

2- Transfect

3- Add fresh media

4- Harvest

5- Analyze

\section{Example (Figure 1).}

1.- Day 1, Split. BAEC cells passage 3-6, 90 \% confluent split 1/4 on 12-well plates. Split one $10 \mathrm{~cm}$ dish or a $75 \mathrm{~cm}^{2}$ flask in 4 12-well plates.

2.- Day 2, transfection with lipofectamine 2000.

2.1 Lipofectamine (Invitrogen) is diluted in culture media without serum or antibiotics $(1 \mu \mathrm{l}$ lipofectamine/70 $\mu \mathrm{l}$ media).

2.2 The DNA pool is diluted $1 \mu \mathrm{g}$ DNA/140 $\mu$ media. Vector concentration should be reduced at least 5x over standard. The DNA pool included per well $250 \mathrm{ng}$ of the luciferase reporter vector (pGL3 in which $2 \mathrm{~kb}$ of the sod2 promoter had been cloned) and the indicated amounts of the TM-FOXO3 plasmid and the corresponding control vector, so that the total DNA amount per well is constant.

2.3 The dilutions are mixed 1:1 and complexes were allowed to form for $20 \mathrm{~min}$ at RT.

2.4 Remove the media from the plates.

2.4 Mix the complexes with fresh cell media $(0.5 \mathrm{ml})$ and then add them to the cells. 
3.- Transfection is allowed to proceed in standard media but in the absence of serum for $6 \mathrm{~h}$. Fresh media with $10 \%$ FBS is then added.

4.- Harvested $24 \mathrm{~h}$ later, the cell culture should be $100 \%$ confluent.

4.1 Each well is washed 3x with cold PBS.

4.2 Add $150 \mu \mathrm{l}$ of lysis buffer to the cells and keep in a rocker on slow motion for for $30 \mathrm{~min}$ at $\mathrm{RT}$.

4.3 Collect the lysate and transfer it to a $1.5 \mathrm{ml}$ microcentrifuge tube. Keep in ice

4.4 Centrifuge to remove cell debrits $1 \mathrm{~min}$ at $13.500 \mathrm{rpm}$ at $4{ }^{0} \mathrm{C}$.

$4.530 \mu \mathrm{l}$ of the lysate is allowed to react with $25 \mu \mathrm{l}$ of luciferase substrate.

4.6 Measure the emitted light in a luminometer, capturing the signal for 2 min.

\subsubsection{Adenoviral Infection}

1.- Preparation of viral stocks.

1.1 Transfect 293A cells with the adenoviral vector use $1 \mu \mathrm{g}$ of DNA to transfect using Lipofectamine 2000 one well (90 \% confluent) in a 6 well plate dish for 4-6 h.

1.2 Do not change the media unless it turns yellow. Harvest the cells by scraping when cytotoxicity becomes evident and before the cells detach from the dish. This should occur about 7-10 days post-transfection, if after 2 weeks in culture the cells do not show sings of viral infection, discard and start again. Wash with cold PBS 2x, scrap the cells from the dish, resuspend in $300 \mu \mathrm{l}$ PBS, lyse the cells using 3 cycles of fast freezing (in liquid N2) and thawing $\left(37^{\circ} \mathrm{C}\right.$ bath), centrifuge for 5 min at $5.000 \mathrm{rpm}$ at $4{ }^{\circ} \mathrm{C}$ to remove cell debrits. Take the supernatant, aliquot it in freezing vials and store at $-80{ }^{\circ} \mathrm{C}$. Evaluation of GFP positive cells using a fluorescence inverted microscope can be used to evaluate transfection efficiency.

1.3 Use $1 / 3$ of the total viral preparation to infect a $3 \mathrm{~cm}$ dish of confluent 293A cells. Gently mix the viral preparation with the cell media and then add it to the cells. Reduce the media volume to a minimun $(1.5 \mathrm{ml})$. Change the media $6 \mathrm{~h}$ later. As in the previous cycle, harvest the cells by scraping when cytotoxicity becomes evident (loss of cell volume, citosol srinkage) and before the cells detach from the plate, now this should occur about 5-7 days post-infection. If the number of days that it 
takes to detect cytotoxicity is not significantly reduced in comparison to the previous amplification cycle, discard. Lyse in $1 \mathrm{ml}$.

1.4 Repeat the infection/harvesting cycle again using $1 / 3$ of the total viral preparation but infecting one $10 \mathrm{~cm}$ cell plate, and then again in a $15 \mathrm{~cm}$ cell plate. At this point the cytotoxicity should be evident about 2 days post infection, if there are no signs after 3 days of infection, discard.

1.5 Use 1/3 of the viral preparation to infect $2015 \mathrm{~cm}$ cell dishes, cytotoxicity should be evident about 2 days post infection, if there are no signs after 3 days of infection, discard. Prepare $5 \mathrm{ml}$ of cell lysate.

1.6 Purify the preparation passing it through a commercially available purification column (ie Cell Biolabs) and following the manufacturer instructions.

1.7 To evaluate the number of infective viral particles (pfu), prepare serial dilutions in PBS up to 10-15 and use them to infect 10 wells of a 96 well cell plate with confluent 293A cells (seeded directly to conflucency and infected 6-12 h later). Count the number of wells with viral halos (clear circles) per dilution to estimate the $\mathrm{pfu} / \mathrm{ml}$. For FOXO viruses, the final viral yield is generally $10^{10}{ }^{11} \mathrm{pfu} / \mathrm{ml}$.

1.8 When you run out of the stock, DO NOT use it to re-amplify de virus. Start again from the previous cycle of viral preparation. Since FOXO viral vectors are highly unstable we avoided serial re-amplification of viral stocks and always used previous cycle preparations or prepared from plasmid vectors to generate new stocks.

1.9 Following the evaluation of the pfu using a standard serial dilution procedure it is highly recommended to test for the level of protein expression. Both primary cells (i.e. BAEC) and cell lines (i.e. EBNA) can be used for Adenoviral (Ad) load testing. As a rule, we tested first on a cell line that is easily infected, that requires 1-2 moi to get all the cells infected, and re-tested to confirm the optimal working conditions on the cell type that is under investigation (BAEC). Using FOXO viruses is highly recommended to use a vector that co-expreses a reporter gene, like GFP and, as indicated above, use the FOXO/GFP ration as a quality control evaluator of the viral preparation.

\section{Example (Figures 2-3).}

Infect confluent cultures in 10\% FBS for $6 \mathrm{~h}$ with a control Ad-GFP virus, the Ad-TMFOXO3 virus and carry on non-infected cells. 
Change to fresh media and let cells recover o/n, change media again and 24-48 h later monitor infection in an inverted fluorescent microscope to evaluate GFP levels and harvest cells. Whole cells extracts were used for WB for GFP, FOXO3 and $\beta$-actin as loading control. GFP controls for viral load.

\subsubsection{Evaluation of FOXO stress resistance activity}

As outlined above, FOXO needs to work in conditions when oxidative metabolism in quiescent confluent cultures to efficiently induce the pro-survival stress resistance programme, because in this conditions the activity of the cofactors that boost its antioxidant activity will be maximized. Therefore, the levels of oxidants will be kept under control, and will not be able to drive cell death. However, it has also to be noted, that once a certain oxidant level is reached, even in this conditions (actually boosted by them), the cell death program will be activated in a FOXO dependent manner. In other words, FOXOs control ROS more efficiently in conditions of nutrient limitation but also drive apoptosis in these conditions once a certain threshold level of oxidants is reached.

As a consequence, to induce antioxidant or stress resistance genes efficiently in a FOXO dependent manner, several procedures can be implemented as indicated above. The simplest one of them being to work with constitutively active proteins in standard culture conditions, but using very short time spans, before the cell death program is activated. Obviously, this will not suffice to evaluate cell death resistance but will provide a robust and highly reproducible gene expression control setting.

Working with a constitutively active mutant. Example (Figure 4).

1.- Infect confluent HUVEC cells for $6 \mathrm{~h}$ with equivalent viral doses of Ad-GFP or AdTM-FOXO3 in triplicates.

2.- Change the media and allow cells to recover $\mathrm{o} / \mathrm{n}$.

3.- Harvest cells prior to WB analysis.

Silencing sh-FOXO vectors provide a more physiological testing setting, however to induce FOXO activity and be able to detect differences in stress resistance gene 
expression, it has to be activated, the simplest procedure being serum deprivation. Since serum deprivation induces cell death, partial deprivation and short experimental time frames are the most convenient setting.

Working with a constitutively active silencing vector. Example (Figure 5).

1.- Infect $\mathrm{o} / \mathrm{n}$ confluent cultures of targeted cells in standard culture media with $10 \%$ FBS with Ad-shControl or Ad-shFOXO3.

2.- In the morning add fresh media with $10 \%$ FBS.

3.- The same evening change the media again, this time the media would be supplemented with just $0.5 \%$ FBS.

4.- Harvest the cells the next morning.

Evaluating the effect of a co-factor.

When evaluating the effect of a co-regulator on FOXO activity we may have to simultaneously modify the levels of the co-regulator and FOXO. Working with viral vectors or transfections means to work with relatively high doses of vectors (double what would be normally required). Since dealing with FOXO in standard culture conditions may result in enhanced cell death, it is highly recommended to establish two step protocols, where the vector for the co-factor is incorporated first.

\section{Example.}

1.- Confluent BAEC cells (p3) in 6 well plates are infected o/n with Ad-Control or AdshSIRT1, aiming to silence the FOXO activator SIRT1 in standard culture media with $10 \%$ FBS.

2.- The following morning fresh media is added supplemented with $10 \%$ FBS and the cells are allowed to recover during the day.

3.- Then, the cells are infected with Ad-control or Ad-shFOXO3 o/n.

4.- The following morning, free media is added supplemented with $0.5 \% \mathrm{FBS}$, 5.- $8 \mathrm{~h}$ later the cells are harvested.

\section{Acknowledgements}


This work was supported by grants from the Spanish "Ministerio de Economía Industria y Competitividad' (MINEICO) and FEDER funds [Grant numbers SAF201563904-R, SAF2015-71521-REDC,] and from the EC H2020 framework programm Grant MSCA-ITN-2016-721236.

\section{Notes}

1. Transfection assays for reporter gene expression. When testing for transcription factor activity using reporter vectors encoding luciferase, it is generally or critical importance to test the dose and time of transfection. Even though this is generally true, we found that it is even more so for FOXO evaluation, to test a wide range of plasmid concentrations and time of response, and it is generally advisable to work with as low levels of FOXO driving plasmid as possible unless it is an inducible vector. Working with low levels of FOXO will prevent cell cycle arrest and will facilitate the testing of FOXO activity in confluent cell cultures where its activity is more readily induced without the concomitant activation of programmed cell death.

2. Every cell type has a different level of antioxidants that this will change the dose of pro-oxidant molecules or level of pro-oxidative insult required to activate FOXO. Testing of the minimal dose that mediates FOXO activation in your cell of choice is highly recommended since that will allow you to follow the capacity of FOXO to respond to the oxidative challenge as well as its functional consequences. When apoptosis is induced simultaneously the impact of the process on redox control is so huge that will prevent any meaningful determination of ROS levels.

3. Oxidative stress induces the acetylation of FOXO and simultaneously activates the deacetylase SIRT1. The capacity of FOXO to induce antioxidant genes in response to increased ROS therefore largely depends on the activity of SIRT1. Since the acetylation of FOXO drives its degradation, to follow FOXO activity in response to oxidative stress it is necessary to identify the time window in which FOXO is acetylated by not yet degraded.

4. FOXO antioxidant activity depends to a large extent on the coactivator PGC-1 $\alpha$ that frequently downregulated or completely inactive in highly proliferative cells such as cancer cells. As a result, working with primary cells is easier to analyze FOXO antioxidant activities. 
5. While working with virus is common to use a concentration that ensures that every cell in the dish is infected, as evaluated by a reporter like GFP. Viral infection per se is stressing your cells, and when you increase simultaneously FOXO activity, that may compromise viability and cell cycle progression, so working with low levels of infection can be actually an advantage.

\section{References}

1. Prieto I, Monsalve M (2017) ROS homeostasis, a key determinant in liver ischemicpreconditioning. Redox Biol 12:1020-1025. doi:10.1016/j.redox.2017.04.036

2. Schmidt-Strassburger U, Schips TG, Maier HJ, Kloiber K, Mannella F, Braunstein KE, Holzmann K, Ushmorov A, Liebau S, Boeckers TM, Wirth T (2012) Expression of constitutively active FoxO3 in murine forebrain leads to a loss of neural progenitors. FASEB J 26 (12):4990-5001. doi:10.1096/fj.12-208587

3. Czymai T, Viemann D, Sticht C, Molema G, Goebeler M, Schmidt M (2010) FOXO3 modulates endothelial gene expression and function by classical and alternative mechanisms. J Biol Chem 285 (14):10163-10178. doi:10.1074/jbc.M109.056663

4. Yu H, Fellows A, Foote K, Yang Z, Figg N, Littlewood T, Bennett M (2018) FOXO3a (Forkhead Transcription Factor O Subfamily Member 3a) Links Vascular Smooth Muscle Cell Apoptosis, Matrix Breakdown, Atherosclerosis, and Vascular Remodeling Through a Novel Pathway Involving MMP13 (Matrix Metalloproteinase 13). Arterioscler Thromb Vasc Biol 38 (3):555-565. doi:10.1161/ATVBAHA.117.310502

5. Storz P (2011) Forkhead homeobox type O transcription factors in the responses to oxidative stress. Antioxid Redox Signal 14 (4):593-605. doi:10.1089/ars.2010.3405

6. Skurk C, Maatz H, Kim HS, Yang J, Abid MR, Aird WC, Walsh K (2004) The Aktregulated forkhead transcription factor FOXO3a controls endothelial cell viability through modulation of the caspase-8 inhibitor FLIP. J Biol Chem 279 (2):1513-1525. doi:10.1074/jbc.M304736200

7. Brunet A, Bonni A, Zigmond MJ, Lin MZ, Juo P, Hu LS, Anderson MJ, Arden KC, Blenis J, Greenberg ME (1999) Akt promotes cell survival by phosphorylating and inhibiting a Forkhead transcription factor. Cell 96 (6):857-868

8. He TC, Zhou S, da Costa LT, Yu J, Kinzler KW, Vogelstein B (1998) A simplified system for generating recombinant adenoviruses. Proc Natl Acad Sci U S A 95 (5):2509-2514

9. Olmos Y, Valle I, Borniquel S, Tierrez A, Soria E, Lamas S, Monsalve M (2009) Mutual dependence of Foxo3a and PGC-1alpha in the induction of oxidative stress genes. J Biol Chem 284 (21):14476-14484. doi:10.1074/jbc.M807397200

10. Borniquel S, Garcia-Quintans N, Valle I, Olmos Y, Wild B, Martinez-Granero F, Soria E, Lamas S, Monsalve M (2010) Inactivation of Foxo3a and subsequent downregulation of PGC-1 alpha mediate nitric oxide-induced endothelial cell migration. Mol Cell Biol 30 (16):4035-4044. doi:10.1128/MCB.00175-10 
11. Olmos Y, Sanchez-Gomez FJ, Wild B, Garcia-Quintans N, Cabezudo S, Lamas S, Monsalve M (2013) SirT1 regulation of antioxidant genes is dependent on the formation of a FoxO3a/PGC-1alpha complex. Antioxid Redox Signal 19 (13):1507-1521. doi:10.1089/ars.2012.4713

12. Papanicolaou KN, Izumiya Y, Walsh K (2008) Forkhead transcription factors and $\begin{array}{lllll}\text { cardiovascular biology. } & \text { Circ } & \text { Res } & 102 & \text { (1):16-31. }\end{array}$ doi:10.1161/CIRCRESAHA.107.164186

13. Aguer C, Gambarotta D, Mailloux RJ, Moffat C, Dent R, McPherson R, Harper ME (2011) Galactose enhances oxidative metabolism and reveals mitochondrial dysfunction in human primary muscle cells. PLoS One 6 (12):e28536. doi:10.1371/journal.pone.0028536

14. Lages YM, Nascimento JM, Lemos GA, Galina A, Castilho LR, Rehen SK (2015) Low oxygen alters mitochondrial function and response to oxidative stress in human neural progenitor cells. PeerJ 3:e1486. doi:10.7717/peerj.1486

15. Tiede LM, Cook EA, Morsey B, Fox HS (2011) Oxygen matters: tissue culture oxygen levels affect mitochondrial function and structure as well as responses to HIV viroproteins. Cell Death Dis 2:e246. doi:10.1038/cddis.2011.128

\section{Figure legends}

Fig. 1. Subconfluent BAEC cells seeded on 12-well plates were transfected with Lipofectamine 2000 for $6 \mathrm{~h}$. The DNA mixture contained per well $250 \mathrm{ng}$ of a luciferase reporter plasmid, containing $2 \mathrm{~Kb}$ of the promoter region of a FOXO3-regulated antioxidant gene (sod2) and 20 ng of a mixture of a TM-FOXO3 vector with its control. Cells were harvested $24 \mathrm{~h}$ post-transfection.

Fig. 2. Confluent EBNA cell cultures were non-infected (C), infected with a GFP control Ad vector (GFP) and with the indicated doses of the tested FOXO3 Ad vector. In the panels A-C three different viral preparations were tested. The preparation on panel A yielded a good level of FOXO- expression, the preparation on panel B was marginally positive for the recombinant protein expression and the preparation on panel C was negative for FOXO3 expression. On panel D two different preparations are compared preparation 1 was positive and preparation 2 negative for FOXO3 levels.

Fig. 3. Confluent BAEC cell cultures were non-infected (C), infected with a GFP control Ad vector (GFP) and with the indicated doses of the tested FOXO Ad vector. The viral concentration of the preparation in panel $B\left(2^{\text {nd }}\right.$ preparation $)$ was markedly 
lower than that of panel A ( $1^{\text {st }}$ preparation) but the FOXO/GFP ratios were found to be similar, so the two preparations were considered to be suitable for experiments.

Fig. 4. 6 well plates of confluent HUVEC cells (p6) were infected with equivalent viral doses of Ad-GFP or Ad-TM-FOXO3a in triplicates for $6 \mathrm{~h}$, media was changed and cells allowed to recover o/n and cells were harvested prior to WB analysis.

Fig. 5. 6-well plates of confluent MEF cells (p2) were infected o/n with three equivalent doses of Ad-shControl or Ad-shFOXO3 in standard media with 10\% FBS, cells were allowed to recover during the day in media supplemented with $10 \% \mathrm{FBS}$, and then the cells were serum deprived o/n (0.5\% FBS) and harvested prior to WB analysis.

Fig. 6. 6-well plates of confluent BAEC cells were infected with equivalent doses of Ad-shControl, ad-shSIRT1 and Ad-shFOXO3 as indicated. Cells were first infected o/n with ad-shControl and Ad-shSIRT1, allowed to recover for $8 \mathrm{~h}$ and then re-infected o/n with Ad-shControl and ad-shFOXO3, serum deprived for 8 h (0.5\% FBS) and harvested prior to WB analysis. 